\title{
Loss-of-function HSD17B13 variants, non-alcoholic steatohepatitis and adverse liver outcomes: Results from a multi-ethnic Asian cohort
}

Yi-Wen Ting ${ }^{1}$, Amanda Shen-Yee Kong ${ }^{1}$, Shamsul Mohd Zain ${ }^{2}$, Wah-Kheong Chan ${ }^{3}$, Hwa-Li Tan ${ }^{2}$, Zahurin Mohamed ${ }^{2}$, Yuh-Fen Pung ${ }^{4}$, and Rosmawati Mohamed ${ }^{3}$

${ }^{1}$ Faculty of Medicine, University of Malaya, Kuala Lumpur; ${ }^{2}$ The Pharmacogenomics Laboratory, Department of Pharmacology, Faculty of Medicine, University of Malaya, Kuala Lumpur; ${ }^{3}$ Gastroenterology and Hepatology Unit, Department of Medicine, Faculty of Medicine, University of Malaya, Kuala Lumpur; ${ }^{4}$ Department of Biomedical Sciences, University of Nottingham (Malaysia Campus), Selangor, Malaysia

Graphical Abstract

\begin{tabular}{|c|c|c|}
\hline $\begin{array}{l}\text { Case-control study } \\
223 \text { consecutive biopsy- } \\
\text { proven NAFLD patients } \\
\text { recruited from year } 2009 \text { - } \\
2014\end{array}$ & $\begin{array}{ll} & \text { Excluded }(n=58) \\
\text { - } & \text { Lost to follow-up }(n=49) \\
& \text { Follow-up }<5 \text { years }(n=9)\end{array}$ & $\begin{array}{ll} & \text { Follow-up study } \\
& \text { until } 31^{\text {st }} \text { of August } 2019 \\
\quad & (165 \text { patients) } \\
\text { - } & \text { Cardiovascular disease } \\
\text { - } & \text { Liver-related } \\
& \text { complications } \\
\text { - } & \text { Extrahepatic malignancy } \\
\text { - } & \text { All-cause mortality }\end{array}$ \\
\hline $\begin{array}{c}\text { HSD17B13 rs72613567 TA } \\
\text { allele and rs6834314 G } \\
\text { allele } \\
\text { Lower risk of NAFLD and } \\
\text { NASH }\end{array}$ & & $\begin{array}{c}\text { HSD17B13 rs72613567 } \\
\text { homozygous TA allele and } \\
\text { rs6834314 homozygous G } \\
\text { allele } \\
\text { Lower incidence of liver- }\end{array}$ \\
\hline
\end{tabular}

\begin{abstract}
Abbreviations:
BMI, body mass index; $\mathrm{Cl}$, confidence interval; FBG, fasting blood glucose; $\mathrm{FLI}$ fatty liver index; GGT, gamma-glutamyl transferase; GWAS, genome-wide association study; HCC, hepatocellular carcinoma; HDL, high-density lipoprotein; HR, hazard ratio; HSD17B13, 17ß-hydroxysteroid dehydrogenase 13; HWE, HardyWeinberg equilibrium; indel, insertion and deletion; LDL, low-density lipoprotein; NAFL, non-alcoholic fatty liver; NAFLD, non-alcoholic fatty liver disease; NASH, non-alcoholic steatohepatitis; $\mathrm{OR}$, odds ratio; PCA, principal component analysis; PNPLA3, patatin-like phospholipase domain-containing 3; SNP, single nucleotide polymorphism; UMMC, University of Malaya Medical Centre
\end{abstract}

Corresponding author : Shamsul Mohd Zain

The Pharmacogenomics Laboratory, Department of Pharmacology, Faculty of Medicine, University of Malaya, Jalan Universiti, Kuala Lumpur 50603, Malaysia

Tel: +60-3-79673195, Fax: +60-3-7967479

E-mail:shamsul@um.edu.my

https://orcid.org/0000-0002-3441-6659

Editor: Jian-Gao Fan, Xinhua Hospital Affiliated to Shanghai Jiaotong University School of Medicine, China 
Background/Aims: 17 $\beta$-hydroxysteroid dehydrogenase 13 (HSD17B13) variants were recently reported to have significantly lower odds of non-alcoholic fatty liver disease (NAFLD). This is a two-part study that aimed to evaluate the association of HSD17B13 variants with NAFLD and its histological severity, and to identify the association of the variants with clinical outcomes in a cohort of biopsy-proven NAFLD patients.

Methods: Consecutive biopsy-proven NAFLD patients and controls without fatty liver were recruited for this study between 2009 and 2014. Genotyping for HSD17B13 variants was performed using rhAmp assays. A total of 165 patients with NAFLD were monitored up until August 2019. Clinical outcomes were recorded.

Results: HSD17B13 rs72613567 TA allele and rs6834314 G allele were associated with lower odds of non-alcoholic steatohepatitis (NASH) in the overall cohort and among ethnic Chinese, but not among ethnic Malays or Indians $(P<0.05)$. During a mean follow-up of 89 months, 32 patients (19.4\%) experienced at least one clinical outcome (cardiovascular events, $n=22$; liver-related complications, $n=6$; extra-hepatic malignancy, $n=5$; and mortality, $n=6$ ). The rs72613567 homozygous TA allele and the rs 6834314 homozygous $\mathrm{G}$ allele were independently associated with a lower incidence of liver-related complications (hazard ratio [HR], 0.004; 95\% confidence interval [Cl], $0.00-0.64 ; P=0.033$ and $\mathrm{HR}, 0.01 ; 95 \%$ $\mathrm{Cl}, 0.00-0.97 ; P=0.048$, respectively) and were associated with lower grade of hepatocyte ballooning among the ethnic Chinese.

Conclusion: HSD17B13 rs72613567 and rs6834314 variants were inversely associated with NAFLD and NASH, and were associated with lower incidence of adverse liver outcomes in a cohort of multi-ethnic Asian patients with NAFLD. (Clin Mol Hepatol 2021;27:486-498)

Keywords: Polymorphism, Genetic; Genetic variation; Liver diseases; Asia; Malaysia

\section{Study Highlights}

This study is unique in that it evaluates the association of HSD17B13 with NAFLD and its clinical outcomes in the Asian population. We assessed the clinical impact of HSD17B13 genetic variants (rs72613567 and rs6834314) by applying a case-control and a longitudinal approach. Loss-of-function HSDI7B13 variants were found to be associated with a lower risk of NAFLD and lower incidence of adverse liver outcomes in a cohort of multi-ethnic Asian patients with NAFLD.

\section{INTRODUCTION}

An adverse liver outcome is one of the leading causes of death in non-alcoholic fatty liver disease (NAFLD). ${ }^{1}$ Non-alcoholic steatohepatitis (NASH) has become the second leading indication for liver transplantation in the United States among patients with or without hepatocellular carcinoma (HCC). ${ }^{2}$ The significant morbidity and mortality related to NAFLD can be attributed to the increase in its prevalence, which parallels the rising prevalence of obesity and metabolic syndrome worldwide. Genetic factors are increasingly recognised in the pathophysiology of this multifactorial disease. ${ }^{3}$

Several studies hypothesised that patatin-like phospholipase domain-containing 3 (PNPLA3) rs738409, the first risk genetic variant discovered from the genome-wide association study (GWAS) of NAFLD, could be utilised as a potential prognostic factor in clinical settings. ${ }^{4,5}$ Recently, 17/-hydroxysteroid dehydrogenase 13 (HSD17B13), a liver-specific lipid-droplet associated pro- tein, was found to act in an opposite manner. A recent landmark study involving 46,544 European and Hispanic descendants reported that HSD17B13 rs72613567, a protein-truncating insertion and deletion (indel) variant, is associated with a lower risk of cirrhosis and $\mathrm{HCC}^{6}$ Similar results were replicated in NAFLD patients of European ancestry and in Argentinian and Danish populations. ${ }^{7-9}$ Another single amino acid mutation at rs6834314 is also associated with the loss of HSD17B13 enzymatic activity. The HSD17B13 rs6834314 variant, which is highly linked with the rs72613567 variant, attenuates the effect of PNPLA3 on advanced liver fibrosis among the Japanese. ${ }^{10}$ Growing evidence has shown the critical role of HSD17B13 in the regulation of hepatic lipid homeostasis, which could potentially represent a new target for the treatment of NAFLD.

This study aimed to evaluate the clinical impact of HSD17B13 genetic variants (rs72613567 and rs6834314) by applying a casecontrol and a longitudinal approach. To the best of our knowledge, this is the first study to evaluate the association of HS- 
D17B13 with NAFLD and its clinical outcomes in the Asian population.

\section{MATERIALS AND METHODS}

\section{Study subjects}

Consecutive biopsy-proven NAFLD patients were recruited from the Gastroenterology and Hepatology Clinic, University of Malaya Medical Centre (UMMC) from 2009 to 2014. Patients with viral hepatitis B or C infection, autoimmune liver diseases, significant alcohol intake ( $>21$ units per week for men and $>14$ units per week for women), and use of medication that can cause steatosis or other causes of chronic liver diseases were excluded from this study. ${ }^{1}$ The controls were healthcare workers, family members, or friends of patients recruited from UMMC during the same period of time. The control subjects had a normal body mass index (BMI) and an ultrasound examination was conducted to exclude presence of fatty liver. They had normal liver function tests, fasting blood glucose (FBG), and lipid profile. The study protocol was approved by the UMMC Medical Research Ethics Committee (MREC Reference No.: 702.11 and ID No.: 2019813-7734) and conformed to the provisions of the Declaration of Helsinki. Written informed consent was obtained from all study participants.

\section{Clinical and laboratory parameters}

Demographic, anthropometric, clinical, and laboratory data were obtained using a standard protocol. Obesity was defined as BMI $\geq 25 \mathrm{~kg} / \mathrm{m}^{2}$. $^{11}$ Central obesity was defined as waist circumference $\geq 90 \mathrm{~cm}$ for men and $\geq 80 \mathrm{~cm}$ for women. ${ }^{12}$ Hypertension was considered present when there was a self-reported history of hypertension, when the subject was on medication, or when the blood pressure was $\geq 130 / 85 \mathrm{mmHg}$. Diabetes mellitus was considered present when there was a self-reported history of diabetes mellitus, when the subject was on medication, or when FBG was $\geq 7.0 \mathrm{mmol} / \mathrm{L}$. Dyslipidaemia was considered present when there was a self-reported history of dyslipidaemia, when the subject was on medication, or when serum total cholesterol was $\geq 5.2$ $\mathrm{mmol} / \mathrm{L}$, serum triglyceride $\geq 1.7 \mathrm{mmol} / \mathrm{L}$, serum high-density lipoprotein $(\mathrm{HDL})<1.0 \mathrm{mmol} / \mathrm{L}$ for men or $<1.3 \mathrm{mmol} / \mathrm{L}$ for women or serum low-density lipoprotein $(\mathrm{LDL}) \geq 3.4 \mathrm{mmol} / \mathrm{L}$. Patients with $\geq 3$ of the following five criteria were diagnosed with metabolic syndrome according to the National Cholesterol Education Pro- gram Adult Treatment Panel III criteria: central obesity, hypertension, hypertriglyceridaemia, low serum $\mathrm{HDL}$, and impaired fasting glucose (FBG $\geq 5.6 \mathrm{mmol} / \mathrm{L}$ ) or diabetes mellitus. ${ }^{12}$

\section{Liver biopsy and histological assessment}

Ultrasound-guided percutaneous liver biopsy was performed using $18 \mathrm{G}$ TERUMO ${ }^{\circledR}$ Hypodermic Needle (Cardinal Health, Dublin, $\mathrm{OH}, \mathrm{USA}$ ). Liver biopsy slides were examined by an experienced histopathologist and reported according to the NASH clinical research network scoring system. ${ }^{13,14}$ Non-alcoholic fatty liver (NAFL) was defined as hepatic steatosis with no or only mild lobular inflammation and no hepatocyte ballooning. NASH was defined as the presence of hepatic steatosis, lobular inflammation and hepatocyte ballooning (at least grade 1 each) with or without fibrosis. Advanced fibrosis was defined as fibrosis stage $\geq 3$.

\section{Genotyping}

The QiAamp DNA Mini Kit (Qiagen, Hilden, Germany) was used to extract genomic DNA from the concentrated white blood cells of the buffy coat with the quality ranging from 1.8-2.0 $\left(\mathrm{OD}_{260} / \mathrm{OD}_{280}\right)$. HSD17B13 rs72613567 and rs6834314 were genotyped using the rhAmp single nucleotide polymorphism (SNP) genotyping assay (Integrated DNA Technologies, Redwood City, CA, USA) on a StepOnePlus Real-Time PCR system (Applied Biosystems, Foster city, CA, USA) according to the manufacturer's protocol. The custom primer sequences for HSD17B13 were as follows: Forward: 5'-GGTGTTCTGTGCTGTACTTACrUTCTG-3'; Reverse: 5'-GTGTTCTGTGCTGTACTTAArCTTCT-3' for rs72613567 and Forward: 5'-AGGTCTGAAGGGGCAGTAATrGGGCA-3'; Reverse: 5'-AGGTCTGAAGGGGCAGTAACrGGGCA-3' for rs6834314. To ensure genotyping confidence, positive and negative controls were introduced for each run. gBlocks Gene Fragments (Integrated DNA Technologies) were used as positive controls. Additional genotyping details can be found in Supplementary Material 1.

\section{Follow-up study}

Only biopsy-proven NAFLD patients with a minimum follow-up of 5 years were included in the longitudinal study. These patients were on a regular 6-monthly follow-up at the gastroenterology and hepatology clinic with blood test \pm ultrasound performed prior to the clinic visit. Their medical records were also carefully studied from the date of the liver biopsy until 31st August 2019 to 
record any cardiovascular events, liver-related complications, malignancy, or mortality. Cardiovascular events included a history of acute myocardial infarction, congestive cardiac failure, history of coronary revascularisation, and stroke. Liver-related complications included ascites, hepatic encephalopathy, spontaneous bacterial peritonitis, history of gastro-oesophageal varices or variceal bleeding, HCC, and hepatorenal syndrome. The cause of malignancy or cause of death was recorded for patients who developed malignancy or died.

\section{Statistical analysis}

Power analysis was carried out using Quanto to calculate the sample size. ${ }^{15} \mathrm{~A}$ total of 143 NAFLD cases and controls were needed to provide a desired power of $80 \%$ at a of 0.05 under a gene-environment model with the following assumptions: the allele frequency ranged from 0.20 to 0.40 , the baseline risk for the Malaysian population was 0.23 and the minimum detectable odds ratio was 2.0. ${ }^{16}$ The genotype distribution was assessed for HardyWeinberg equilibrium (HWE) using a goodness-of-fit chi-squared test with one degree of freedom. $P$-value of more than 0.05 indicates an agreement with the equilibrium. Patients with incomplete genotyping data were excluded from data analysis. Data analyses were carried out using SPSS ver. 23.0 (IBM Corp., Chicago, IL, USA). Values were presented as mean \pm standard deviation for continuous variables while categorical variables were recorded as number and percentages. All variables were tested for normality using Kolmogorov-Smirnov test. For group comparison, Student's $t$-test was used for normally distributed variables whereas Mann-Whitney U-test was used for skewed variables. Allelic association was identified using logistic regression and multiple logistic regression was performed after adjusting for age and gender. Kruskal-Wallis test was used to compare medians between three groups for skewed variables. Jonckheere-Terpstra test was conducted for univariate analysis of the association between genotype and liver histological grading. Multivariate ordinal regression analysis was performed after adjusting for other histological features. Clinical outcomes were analysed using Kaplan-Meier survival curves and compared between NAFLD patients with different HSD17B13 genotyping status. Cox proportional-hazards regression analysis was conducted for analyses of clinical outcome risks and its association with each genotype. Different genetic models were fitted into analysis for better understanding of the inheritance effect. Principal component analysis (PCA) was performed to verify the results. The number of factors were selected based on the criteria of eigenvalue $>1.0$. An orthogonal varimax rotational was used to produce interpretable factors. A total of four factors were extracted by PCA. The Kaiser-Meyer-Olkin measure of sampling adequacy is over 0.50 . Factor 1 contained age and gender, factor 2 contained hypertension and dyslipidaemia status on follow-up, factor 3 showed high loadings for liver outcome and serum gamma-glutamyl transferase (GGT) level and factor 4 contained HSD17B13 variant and advanced fibrosis status. Results were considered statistically significant at $P$ value of $<0.05$.

\section{RESULTS}

\section{Case-control study of HSD17B13 variants in patients with biopsy-proven NAFLD}

A total of 223 biopsy-proven NAFLD patients and 205 controls were included in the case-control study. The characteristics of the subjects are shown in Table 1. The allele frequencies and association tests of HSD17B13 rs72613567 and rs6834314 in NAFLD patients compared with those of the controls are presented in Table 2. The genotype distribution was in HWE for all patients and controls. Overall, rs72613567 TA allele frequency was lower among patients with NAFLD compared with that in controls (adjusted odds ratio [OR], 0.59; 95\% confidence interval [Cl], 0.40-0.88; $P=0.009)$, indicating a protective effect $(O R<1)$ of this allele against the disease. Further analysis stratified by ethnicity found the association to be significant among ethnic Chinese $(P<0.05)$ but not among ethnic Malays and Indians $(P>0.05)$. Patients with NASH had lower TA allele frequency compared with that of the controls (adjusted OR, 0.55; 95\% Cl, 0.36-0.83; $P=0.005$ ). Further analysis stratified by ethnicity found the association to be significant among ethnic Chinese $(P<0.05)$ but not among ethnic Malays and Indians $(P>0.05)$. No significant difference was observed in TA allele frequency in patients with NAFL compared with that of the controls $(P>0.05)$. Further analysis stratified by ethnicity found that the TA allele frequency was associated with lower odds of NASH among biopsy-proven NAFLD patients only among ethnic Chinese individuals $(P<0.05)$, but not among ethnic Malays and Indians $(P>0.05)$. Similar results were found for the rs6834314 $\mathrm{G}$ allele. Both genetic variants fit the dominant genetic model $(P<0.05$; Supplementary Table 1).

The characteristics of biopsy-proven NAFLD patients with different HSD17B13 rs72613567 and rs6834314 genotypes can be 


\section{CLINICAL and MOLECULAR}

Volume_27 Number_3 July 2021

Table 1. Characteristics of biopsy-proven NAFLD patients compared with controls

\begin{tabular}{|c|c|c|c|}
\hline & $\begin{array}{l}\text { Biopsy-proven NAFLD } \\
\text { patients }(n=223)\end{array}$ & $\begin{array}{l}\text { Controls } \\
(n=205)\end{array}$ & $P$-value \\
\hline Age (years) & $55.6 \pm 12.5$ & $51.0 \pm 12.5$ & $<0.001$ \\
\hline Male & $117(52.5)$ & $87(42.4)$ & 0.038 \\
\hline Ethnicity & & & 0.021 \\
\hline Malay & $113(50.7)$ & $71(34.6)$ & \\
\hline Chinese & $61(27.4)$ & $86(42.0)$ & \\
\hline Indian & $49(22.0)$ & $48(23.4)$ & \\
\hline BMI $\left(\mathrm{kg} / \mathrm{m}^{2}\right)$ & $29.0 \pm 4.7$ & $22.0 \pm 2.2$ & $<0.001$ \\
\hline HbA1c (\%) & $6.5 \pm 1.5$ & $5.5 \pm 0.5$ & $<0.001$ \\
\hline Total cholesterol (mmol/L) & $5.1 \pm 1.1$ & $4.6 \pm 0.7$ & $<0.001$ \\
\hline Triglyceride (mmol/L) & $1.7 \pm 0.7$ & $1.2 \pm 0.4$ & $<0.001$ \\
\hline $\mathrm{HDL}(\mathrm{mmol} / \mathrm{L})$ & $1.2 \pm 0.3$ & $1.3 \pm 0.4$ & $<0.001$ \\
\hline LDL (mmol/L) & $3.2 \pm 1.0$ & $2.3 \pm 0.5$ & $<0.001$ \\
\hline ALT (U/L) & $81.4 \pm 46.8$ & $33.8 \pm 17.4$ & $<0.001$ \\
\hline $\mathrm{AST}(\mathrm{U} / \mathrm{L})$ & $45.6 \pm 25.9$ & $22.1 \pm 9.3$ & $<0.001$ \\
\hline GGT (U/L) & $106.8 \pm 106.3$ & $41.2 \pm 26.0$ & $<0.001$ \\
\hline \multicolumn{4}{|l|}{ Liver histological features } \\
\hline \multicolumn{4}{|l|}{ Steatosis } \\
\hline 0 & $0(0.0)$ & & \\
\hline 1 & $65(29.1)$ & & \\
\hline 2 & $103(46.2)$ & & \\
\hline 3 & $55(24.7)$ & & \\
\hline \multicolumn{4}{|l|}{ Lobular inflammation } \\
\hline 0 & $7(3.1)$ & & \\
\hline 1 & $126(56.5)$ & & \\
\hline 2 & $86(38.6)$ & & \\
\hline 3 & $4(1.8)$ & & \\
\hline \multicolumn{4}{|l|}{ Hepatocyte ballooning } \\
\hline 0 & $37(16.6)$ & & \\
\hline 1 & $125(56.1)$ & & \\
\hline 2 & $61(27.4)$ & & \\
\hline $\mathrm{NASH}$ & $175(78.5)$ & & \\
\hline \multicolumn{4}{|l|}{ Fibrosis } \\
\hline 0 & $50(22.4)$ & & \\
\hline 1 & $89(39.9)$ & & \\
\hline 2 & $43(19.3)$ & & \\
\hline 3 & $36(16.1)$ & & \\
\hline 4 & $5(2.2)$ & & \\
\hline
\end{tabular}

Values are presented as mean \pm standard deviation or number $(\%)$.

NAFLD, non-alcoholic fatty liver disease; BMI, body mass index; HbA1C, haemoglobin A1c; HDL, high-density lipoprotein; LDL, low-density lipoprotein; ALT, alanine aminotransferase; AST, aspartate aminotransferase; GGT, gamma-glutamyl transferase; NASH, non-alcoholic steatohepatitis. 
Yi-Wen Ting, et al.

Loss-of-function HSD17B13 variants

Table 2. The HSD17B13 rs72613567 and rs6834314 allele frequencies and association tests in biopsy-proven NAFLD patients compared with controls

\begin{tabular}{|c|c|c|c|c|c|}
\hline & Allele frequency & OR $(95 \% \mathrm{Cl})$ & $P$-value & Adjusted OR (95\% CI) & $P$-value \\
\hline \multicolumn{6}{|c|}{ rs72613567 TA allele } \\
\hline \multicolumn{6}{|c|}{ NAFLD vs. control } \\
\hline Overall & 0.24 vs. 0.34 & $0.58(0.40-0.86)$ & 0.006 & $0.59(0.40-0.88)$ & 0.009 \\
\hline Malays & 0.28 vs. 0.40 & $0.60(0.33-1.09)$ & 0.092 & $0.62(0.34-1.13)$ & 0.117 \\
\hline Chinese & 0.26 vs. 0.41 & $0.41(0.21-0.81)$ & 0.010 & $0.31(0.15-0.67)$ & 0.003 \\
\hline Indians & 0.11 vs. 0.14 & $0.78(0.31-1.97)$ & 0.597 & $0.71(0.27-1.87)$ & 0.492 \\
\hline \multicolumn{6}{|c|}{ NAFL vs. control } \\
\hline Overall & 0.27 vs. 0.34 & $0.73(0.39-1.37)$ & 0.330 & $0.72(0.38-1.38)$ & 0.326 \\
\hline Malays & 0.22 vs. 0.40 & $0.45(0.18-1.11)$ & 0.081 & $0.49(0.20-1.24)$ & 0.132 \\
\hline Chinese & 0.50 vs. 0.41 & $1.98(0.51-7.71)$ & 0.327 & $1.81(0.44-7.53)$ & 0.414 \\
\hline Indians & 0.06 vs. 0.14 & $0.39(0.04-3.44)$ & 0.392 & $0.30(0.03-2.98)$ & 0.303 \\
\hline \multicolumn{6}{|c|}{ NASH vs. control } \\
\hline Overall & 0.23 vs. 0.34 & $0.55(0.36-0.83)$ & 0.004 & $0.55(0.36-0.83)$ & 0.005 \\
\hline Malays & 0.31 vs. 0.40 & $0.65(0.35-1.23)$ & 0.187 & $0.67(0.35-1.28)$ & 0.224 \\
\hline Chinese & 0.20 vs. 0.41 & $0.27(0.13-0.57)$ & 0.001 & $0.19(0.08-0.45)$ & $<0.001$ \\
\hline Indians & 0.12 vs. 0.14 & $0.87(0.33-2.26)$ & 0.772 & $0.83(0.31-2.24)$ & 0.708 \\
\hline \multicolumn{6}{|c|}{ NASH vs. NAFL } \\
\hline Overall & 0.23 vs. 0.27 & $0.75(0.39-1.43)$ & 0.384 & $0.75(0.39-1.44)$ & 0.386 \\
\hline Malays & 0.31 vs. 0.22 & $1.46(0.61-3.49)$ & 0.402 & $1.51(0.62-3.67)$ & 0.368 \\
\hline Chinese & 0.20 vs. 0.50 & $0.14(0.03-0.57)$ & 0.006 & $0.13(0.03-0.57)$ & 0.007 \\
\hline Indians & 0.12 vs. 0.06 & $2.26(0.25-20.65)$ & 0.471 & $2.25(0.23-22.13)$ & 0.488 \\
\hline \multicolumn{6}{|c|}{ rs6834314 G allele } \\
\hline \multicolumn{6}{|c|}{ NAFLD vs. control } \\
\hline Overall & 0.26 vs. 0.39 & $0.59(0.40-0.86)$ & 0.006 & $0.59(0.40-0.87)$ & 0.009 \\
\hline Malays & 0.31 vs. 0.44 & $0.65(0.36-1.20)$ & 0.171 & $0.66(0.36-1.22)$ & 0.189 \\
\hline Chinese & 0.29 vs. 0.45 & $0.39(0.20-0.77)$ & 0.006 & $0.31(0.14-0.66)$ & 0.002 \\
\hline Indians & 0.12 vs. 0.19 & $0.71(0.29-1.74)$ & 0.458 & $0.64(0.25-1.64)$ & 0.355 \\
\hline \multicolumn{6}{|c|}{ NAFL vs. control } \\
\hline Overall & 0.29 vs. 0.39 & $0.79(0.42-1.48)$ & 0.453 & $0.74(0.38-1.47)$ & 0.391 \\
\hline Malays & 0.26 vs. 0.44 & $0.54(0.22-1.31)$ & 0.173 & $0.60(0.24-1.49)$ & 0.267 \\
\hline Chinese & 0.50 vs. 0.45 & $2.52(0.52-12.15)$ & 0.250 & $2.26(0.44-11.61)$ & 0.329 \\
\hline Indians & 0.06 vs. 0.19 & $0.31(0.04-2.79)$ & 0.299 & $0.20(0.02-1.90)$ & 0.160 \\
\hline \multicolumn{6}{|c|}{ NASH vs. control } \\
\hline Overall & 0.26 vs. 0.39 & $0.54(0.36-0.82)$ & 0.003 & $0.48(0.31-0.75)$ & 0.001 \\
\hline Malays & 0.33 vs. 0.44 & $0.70(0.37-1.32)$ & 0.270 & $0.71(0.37-1.35)$ & 0.291 \\
\hline Chinese & 0.23 vs. 0.45 & $0.25(0.12-0.53)$ & $<0.001$ & $0.20(0.09-0.45)$ & $<0.001$ \\
\hline Indians & 0.13 vs. 0.19 & $0.81(0.32-2.03)$ & 0.648 & $0.76(0.29-2.00)$ & 0.582 \\
\hline \multicolumn{6}{|c|}{ NASH vs. NAFL } \\
\hline Overall & 0.26 vs. 0.29 & $0.69(0.36-1.31)$ & 0.256 & $0.69(0.36-1.32)$ & 0.263 \\
\hline Malays & 0.33 vs. 0.26 & $1.30(0.55-3.09)$ & 0.555 & $1.37(0.57-3.30)$ & 0.485 \\
\hline Chinese & 0.23 vs. 0.50 & $0.10(0.02-0.50)$ & 0.005 & $0.10(0.02-0.50)$ & 0.006 \\
\hline Indians & 0.13 vs. 0.06 & $2.57(0.28-23.31)$ & 0.402 & $2.29(0.23-22.48)$ & 0.476 \\
\hline
\end{tabular}

NAFL was defined as hepatic steatosis with no or only mild lobular inflammation and no hepatocyte ballooning. NASH was defined as the presence of hepatic steatosis, lobular inflammation and hepatocyte ballooning (at least grade 1 each) with or without fibrosis.

Logistic regression was used to obtain OR. The adjusted OR was adjusted for age and gender.

Control was the reference group in analysis NAFLD vs. control, NAFL vs. control and NASH vs. control. NAFL was used as the reference group in analysis for NASH vs. NAFL.

HSD17B13, 17ß-hydroxysteroid dehydrogenase 13; NAFLD, non-alcoholic fatty liver disease; OR, odds ratio; Cl, confidence interval; NAFL, non-alcoholic fatty liver; NASH, non-alcoholic steatohepatitis. 


\section{CLINCAL and MOLECULAR}

Table 3. Demographic and clinical characteristics of NAFLD patients with a minimum follow-up of 5 years $(n=165)$

\begin{tabular}{|c|c|}
\hline Variable & NAFLD cases $(n=165)$ \\
\hline Age (years) & $58.9 \pm 10.4$ \\
\hline Gender, male & $81(49.1)$ \\
\hline \multicolumn{2}{|l|}{ Ethnicity } \\
\hline Malay & $80(48.5)$ \\
\hline Chinese & $47(28.5)$ \\
\hline Indian & $38(23.0)$ \\
\hline Diabetes mellitus & $108(65.5)$ \\
\hline Hypertension & $117(70.9)$ \\
\hline Dyslipidaemia & $153(92.7)$ \\
\hline Metabolic syndrome & $131(79.4)$ \\
\hline BMI $\left(\mathrm{kg} / \mathrm{m}^{2}\right)$ & $28.9 \pm 4.9$ \\
\hline Waist circumference (cm) & $98.1 \pm 11.1$ \\
\hline Male & $99.9 \pm 10.7$ \\
\hline Female & $95.4 \pm 10.2$ \\
\hline \multicolumn{2}{|l|}{ Blood pressure } \\
\hline Systolic (mmHg) & $135.7 \pm 14.4$ \\
\hline Diastolic (mmHg) & $78.5 \pm 10.4$ \\
\hline Fasting blood glucose (mmol/L) & $6.6 \pm 2.1$ \\
\hline HbA1c (\%) & $7.1 \pm 1.6$ \\
\hline Total Cholesterol (mmol/L) & $4.6 \pm 1.1$ \\
\hline Triglyceride (mmol/L) & $1.7 \pm 0.9$ \\
\hline $\mathrm{HDL}(\mathrm{mmol} / \mathrm{L})$ & $1.3 \pm 0.3$ \\
\hline LDL (mmol/L) & $2.6 \pm 1.0$ \\
\hline Albumin (mg/L) & $38.8 \pm 5.5$ \\
\hline Total bilirubin $(\mu \mathrm{mol} / \mathrm{L})$ & $13.3 \pm 8.7$ \\
\hline $\operatorname{ALT}(U / L)$ & $47.8 \pm 33.5$ \\
\hline AST (U/L) & $44.7 \pm 54.1$ \\
\hline $\operatorname{ALP}(U / L)$ & $87.8 \pm 77.5$ \\
\hline GGT (U/L) & $94.0 \pm 149.3$ \\
\hline Platelet $\left(10^{9} / \mathrm{L}\right)$ & $293.0 \pm 216.9$ \\
\hline \multicolumn{2}{|l|}{ Baseline liver histological features } \\
\hline \multicolumn{2}{|l|}{ Steatosis } \\
\hline 0 & $0(0.0)$ \\
\hline 1 & $40(24.3)$ \\
\hline 2 & $84(50.9)$ \\
\hline 3 & $41(24.8)$ \\
\hline \multicolumn{2}{|l|}{ Lobular inflammation } \\
\hline 0 & $4(2.4)$ \\
\hline 1 & $90(54.5)$ \\
\hline 2 & $68(41.2)$ \\
\hline
\end{tabular}

Table 3. Continued

\begin{tabular}{lc}
\hline Variable & NAFLD cases $(\mathbf{n}=\mathbf{1 6 5})$ \\
\hline 3 & $3(1.8)$ \\
Hepatocyte ballooning & \\
0 & $28(17.0)$ \\
1 & $89(53.9)$ \\
2 & $48(29.1)$ \\
NASH & $129(78.2)$ \\
Fibrosis & \\
0 & $34(20.6)$ \\
1 & $68(41.2)$ \\
2 & $29(17.6)$ \\
3 & $30(18.2)$ \\
4 & $4(2.4)$ \\
HSD17B13 rs72613567 genotype & \\
T/T & $94(57.0)$ \\
T/TA & $55(33.3)$ \\
TA/TA & $16(9.7)$ \\
HSD17B13 rs6834314 genotype & \\
A/A & $89(53.9)$ \\
A/G & $63(38.2)$ \\
G/G & $13(7.9)$ \\
Vaes & \\
\hline & \\
\hline &
\end{tabular}

Values are presented as mean \pm standard deviation or number (\%).

NAFLD, non-alcoholic fatty liver disease; BMI, body mass index; HbA1C, haemoglobin A1c; HDL, high-density lipoprotein; LDL, low-density lipoprotein; ALT, alanine aminotransferase; AST, aspartate aminotransferase; ALP, alkaline phosphatase; GGT, gamma-glutamyl transferase; NASH, non-alcoholic steatohepatitis; HSD 17B13, 17ß-hydroxysteroid dehydrogenase 13.

found in Supplementary Tables 2 and 3. The frequency of the TA and $\mathrm{G}$ alleles was lower among the ethnic Indians compared with that among the ethnic Malays and Chinese. No significant associations were seen between both variants with individual liver histological features using rank-based non-parametric, and regression tests (Supplementary Table 4). Both HSD17B13 rs72613567 T/TA and TA/TA, rs6834314 A/G and G/G genotypes were associated with lower grade of hepatocyte ballooning among the ethnic Chinese $(P<0.05)$.

\section{Follow-up study on clinical outcomes}

One hundred and sixty-five biopsy-proven NAFLD patients had follow-up data for at least 5 years and were included in the outcome analysis. A total of 58 patients were excluded from the outcome analysis (49 patients who were lost to follow-up and nine 
patients had follow-up of $<5$ years). The characteristics of the subjects are summarised in Table 3. The mean age of the study population was $58.9 \pm 10.4$ years old and $49.1 \%$ of them were male. Majority (79.4\%) of patients had metabolic syndrome, while diabetes mellitus, hypertension, and dyslipidaemia were present in $65.5 \%, 70.9 \%$, and $92.7 \%$, respectively. NASH was present in $78.2 \%$ of patients, while advanced fibrosis was present in $20.6 \%$ of cases.

The mean duration of follow-up for the study subjects was 89 months (range, 60-118), corresponding to a total follow-up of 1,227 person-years. During follow-up, 32 patients (19.4\%) experienced at least one clinical outcome. Cardiovascular events topped the list with 22 patients (13.3\%) followed by liver-related compli- cations in six patients (3.6\%). Five patients (3.0\%) developed extrahepatic malignancy. Six patients (3.6\%) died during the followup period; three were due to extrahepatic malignancy, two were liver-related deaths, and one was due to infection. A detailed breakdown of the clinical outcomes according to the genetic variants is shown in Supplementary Table 5.

\section{HSD17B13 variants and clinical outcomes}

The log-rank test revealed no difference in the survival probability of patients with different HSD17B13 genotyping status (Supplementary Figs. 1 and 2). To further investigate the impact of genetic variants on the clinical outcomes of NAFLD, we per-

Table 4. Association of HSD17B13 rs72613567 and rs6834314 genotypes with liver-related complications by Cox regression analysis

\begin{tabular}{|c|c|c|c|c|}
\hline \multirow{2}{*}{ Variable } & Univariate analysis & \multirow{2}{*}{$P$-value } & Multivariate analysis & \multirow{2}{*}{$P$-value } \\
\hline & $\mathrm{HR}(95 \% \mathrm{Cl})$ & & HR $(95 \%$ Cl) & \\
\hline \multicolumn{5}{|l|}{ rs72613567 } \\
\hline $\mathrm{T} / \mathrm{T}$ (reference) & - & - & - & - \\
\hline T/TA & $0.42(0.05-3.73)$ & 0.434 & $0.12(0.01-2.17)$ & 0.152 \\
\hline $\mathrm{TA} / \mathrm{TA}$ & $1.11(0.12-10.01)$ & 0.926 & $0.004(0.00-0.64)$ & 0.033 \\
\hline Age & $1.04(0.95-1.13)$ & 0.418 & - & - \\
\hline Gender & $0.47(0.09-2.58)$ & 0.387 & - & - \\
\hline Advanced fibrosis & $8.94(1.63-48.94)$ & 0.012 & $7.98(1.54-41.50)$ & 0.014 \\
\hline Serum GGT & $1.003(1.002-1.005)$ & $<0.001$ & $1.01(1.00-1.02)$ & 0.010 \\
\hline Diabetes mellitus & $\operatorname{lnf}(N A)$ & 0.315 & - & - \\
\hline Hypertension & $1.77(0.21-15.18)$ & 0.603 & - & - \\
\hline Dyslipidaemia & $0.35(0.04-3.04)$ & 0.344 & - & - \\
\hline BMI & $1.05(0.89-1.23)$ & 0.550 & - & - \\
\hline \multicolumn{5}{|l|}{ rs6834314 } \\
\hline A/A (reference) & - & - & - & - \\
\hline$A / G$ & $0.97(0.16-5.83)$ & 0.977 & $0.46(0.06-3.66)$ & 0.462 \\
\hline $\mathrm{G} / \mathrm{G}$ & $1.60(0.17-15.49)$ & 0.685 & $0.01(0.00-0.97)$ & 0.048 \\
\hline Age & $1.04(0.95-1.13)$ & 0.418 & - & - \\
\hline Gender & $0.47(0.09-2.58)$ & 0.387 & - & - \\
\hline Advanced fibrosis & $8.94(1.63-48.94)$ & 0.012 & $8.93(1.66-47.90)$ & 0.011 \\
\hline Serum GGT & $1.003(1.002-1.005)$ & $<0.001$ & $1.01(1.00-1.02)$ & 0.003 \\
\hline Diabetes mellitus & $\operatorname{lnf}(N A)$ & 0.315 & - & - \\
\hline Hypertension & $1.77(0.21-15.18)$ & 0.603 & - & - \\
\hline Dyslipidaemia & $0.35(0.04-3.04)$ & 0.344 & - & - \\
\hline $\mathrm{BMI}$ & $1.05(0.89-1.23)$ & 0.550 & - & - \\
\hline
\end{tabular}

Inf (NA) represented number was too large to be recorded.

HSD17B13, 17ß-hydroxysteroid dehydrogenase 13; HR, hazard ratio; Cl, confidence interval; GGT, gamma-glutamyl transferase; BMI, body mass index. 
formed Cox regression analysis. Genetic variants and variables with $P \leq 0.10$ in univariate analysis were included in the multivariate analysis. Table 4, Supplementary Tables 6 and 7 show a detailed Cox regression analysis for the association of the genotypes and clinical outcomes.

The rs72613567 T/TA genotype was associated with a higher incidence of cardiovascular outcome (hazard ratio [HR], 3.57; 95\% $\mathrm{Cl}, 1.34-9.48 ; P=0.011)$. Serum HDL cholesterol and systolic blood pressure were independent clinical parameters of cardiovascular outcome $(P<0.05)$ for both rs72613567 and rs6834314.

Serum GGT level was the only independent predictor of extrahepatic malignancy and all-cause mortality.

\section{HSD17B13 variants protected against liver-related complications in NAFLD}

Multivariate Cox regression analysis revealed that the rs 72613567 homozygous TA allele and the rs $6834314 \mathrm{G} / \mathrm{G}$ genotype were associated with a reduced risk of liver-related complications ( $\mathrm{HR}, 0.004 ; 95 \% \mathrm{Cl}, 0-0.64 ; P=0.033$ and $\mathrm{HR}, 0.01 ; 95 \%$ $\mathrm{Cl}, 0-0.97 ; P=0.048$, respectively) but not for cardiovascular events, extrahepatic malignancy, and all-cause mortality (Table 4). Reduced risk of liver-related complications was also observed in the co-dominant model of rs72613567 TA/TA vs. T/T (HR, 0.002; $95 \% \mathrm{Cl}, 0.00-1.23 ; P=0.048$; Supplementary Table 8). Although it did not reach a significant level but close to, there was a suggestive protective trend particularly when the effective alleles were in the homozygous form, as seen in the co-dominant and recessive model (rs72613567 TA/TA and rs6834314 G/G). Advanced fibrosis and serum GGT level were also significant predictors of liver-related complications $(P<0.05)$. The PCA results showed that all components in the four factors explained $60 \%$ of the total variance. Factor 3, which contained serum GGT level and liver-related complications, explained $84 \%$ of the total variance, whereas factor 4, which contained the HSD17B13 variant and advanced fibrosis status, explained $30 \%$ of the total variance.

\section{DISCUSSION}

In this study of a well-characterised cohort of multi-ethnic Asian biopsy-proven NAFLD patients, we found loss-of-function HSD17B13 variants, namely rs72613567 TA allele and rs6834314 G allele, to be inversely associated with NAFLD and NASH in the overall subjects and among ethnic Chinese, but not ethnic Malays and Indians. This study also revealed that the rs72613567 homozygous TA allele and the rs6834314 G/G genotype are associated with reduced risk of liver-related complications and are associated with lower grade of hepatocyte ballooning among the ethnic Chinese.

These novel findings highlight the different impacts of the same genetic variants in persons of different ethnic backgrounds and complements previous interesting observations in genetic studies of NAFLD. Browning and colleagues were the first to report on ethnic differences in the prevalence of significant hepatic steatosis in their landmark paper. ${ }^{17}$ Although Hispanics and Blacks had a similar prevalence of obesity and insulin resistance, the prevalence of significant hepatic steatosis was much higher among Hispanics. The PNPLA3 gene polymorphism was identified and reported as the underlying cause of the observed ethnic differences in their paper that followed 4 years later. ${ }^{4}$ Since then, many subsequent studies have confirmed the importance of this genetic variant in NAFLD. The PNPLA3 genetic polymorphism was not only associated with a higher prevalence of NAFLD but also with more severe liver disease. ${ }^{18}$ Studies on multi-ethnic Malaysian populations have consistently shown a higher prevalence of NAFLD among ethnic Malays and Indians compared with that of ethnic Chinese, and this ethnic predilection can be observed as early as young adulthood. ${ }^{16,19}$ However, interestingly, the frequency of the PNPLA3 risk allele is relatively high among ethnic Chinese compared with that of the other ethnic groups. ${ }^{20} \mathrm{~A}$ meta-analysis has similarly reported high frequencies of the PNPLA3 risk allele among East Asians but a relatively lower prevalence of significant hepatic steatosis. ${ }^{21}$

We set out to determine if the loss-of-function HSD17B13 variants, recently reported to be protective against liver disease, underlining the lower prevalence of NAFLD despite a relatively high frequency of the PNPLA3 risk allele among ethnic Chinese in our multi-ethnic population. We found that HSD17B13 is protective against NAFLD and NASH among ethnic Chinese, but interestingly, not among ethnic Malays and Indians. A recent study among Japanese patients with NAFLD reported an attenuated effect of PNPLA3 on advanced hepatic fibrosis by HSD17B13 rs6834314 G allele. ${ }^{10}$ Abul-Husn et al. ${ }^{6}$ also reported that the HSD17B13 rs72613567-TA allele is associated with decreased PNPLA3 mRNA expression in an allele dosage-dependent manner. Further research is needed to identify the association between both PNPLA3 and HSD17B13 genotypes and their effect on patients with NAFLD.

rs72613567 is an insertion of an adenine adjacent to the donor 
Yi-Wen Ting, et al. Loss-of-function HSD17B13 variants

splice site of exon 6 of HSD17B13 in high linkage with the rs6834314, which is located downstream of the HSD17B13 gene. For instance, rs72613567 T/TA and TA/TA are indel variants which produce truncated protein with reduced enzymatic activity. HSD17B13 was first described as a liver specific lipid-droplet associated protein by Horiguchi et al. ${ }^{22}$ and upregulation of the gene expression in mice and patients with NAFLD was later shown. ${ }^{23}$ HSD17B13 encodes hydroxysteroid 17- $\beta$ dehydrogenase, a protein that plays a pivotal role in hepatic lipid metabolism and overexpression of this enzyme contributes to the development of NAFLD. ${ }^{24}$ Hence, disruption of the protein expression that occurs in loss-of-function variants is a mechanism postulated to mediate its protective effect against liver damage. Support for the finding that HSD17B13 is72613567 is a protective NAFLD gene variant comes from a recent landmark study involving exome-wide sequencing of 46,544 European descents which reported a substantial decrease in serum alanine aminotransferase and aspartate aminotransferase levels associated with the variant. ${ }^{6}$ The study also demonstrated that the variant is associated with a lower risk of NAFLD, as well as non-alcoholic cirrhosis in an allele dose-dependent manner. ${ }^{6}$ The study has pioneered similar independent studies that confirmed the protective effect of the variant against NAFLD. ${ }^{7.8}$ In this study, we showed that the HSD17B13 is72613567TA allele is associated with lower odds of NASH in comparison with the controls, which is in line with the previous findings. ${ }^{6.8} \mathrm{Pi}$ rola et al. ${ }^{7}$ described how the rs72613567-TA allele is significantly associated with decreased levels of hepatic HSD17B13 protein in NAFLD patients bearing the heterozygous T/TA and homozygous TA/TA in an allele dose-dependent manner. Additionally, we replicated similar results in rs6834314, showing that patients bearing the minor $\mathrm{G}$ allele have a reduced risk of NASH. The rs 6834314 variant also demonstrates lower protein levels essential for lipid droplet targeting, which is crucial for protein stability and protection from degradation. ${ }^{8}$ However, as mentioned before, the protective effect of the $T A$ and $G$ alleles was seen only among ethnic Chinese, but not among ethnic Malays and Indians. The cause for this novel finding is uncertain, it may be due to the effect of other genetic variants, and requires further investigation. It is interesting to note that the control frequencies for both rs72613567-TA and rs6834314-G alleles of the East Asian population from the 1000 Genomes Consortium were 0.34 , which are similar to our population at 0.34 and 0.39 , respectively.

Another highlight of our study is that the loss-of-function HSD17B13 variants were found to be associated with reduced risk of liver-related complications, suggesting that the loss-of-function variant may contribute to its protective effect in NAFLD patients. Although there was no significant association between both variants with fibrosis, advanced fibrosis is an independent predictor of liver-related complications. The protective role of the HSD17B13 variants may be lost once advanced fibrosis develops. A recent meta-analysis by Wang et al. ${ }^{25}$ showed that HSD17B13 rs72613567 is associated with significant liver disease protection among NAFLD patients and milder disease severity among studies that included liver histology.

There was a lack of observed association between the TA allele of rs72613567 and steatosis, which is consistent with the findings from previous studies. ${ }^{6,7}$ We also observed no interesting finding on the association between the variant and serum lipid levels. In addition, the expression of the protein on the lipid droplet membrane without affecting the intracellular fat content could potentially explain the lack of association between the TA allele and steatosis. In vitro data reported by Ma and colleagues ${ }^{8}$ lend support to this postulation, whereby overexpressed HSD17B13 in hepatocytes does not affect their fat-storage capacity. Thus, it seems reasonable to presume that HSD17B13 does not modulate lipid content in a direct manner and the variant plays a much more important role in the pathogenesis of liver injury. However, the splice variant was shown to protect against liver ballooning degeneration, lobular inflammation and fibrosis. ${ }^{7}$ Similarly, in this study, we have demonstrated that the loss-of-function HSD17B13 variants are associated with lower grade of hepatocyte ballooning among the ethnic Chinese. Although HSD17B13 has been discovered as a liver specific lipid-droplet associated enzyme which contributes to the pathogenesis of NAFLD, no firm conclusions can be drawn regarding the underlying mechanism on how the change in HSD17B13 activity modulates the liver lipid homeostasis due to its unknown substrates and metabolites.

A recent genetic association study in Japan reported that the carriage of the rs6834314 $\mathrm{G}$ allele attenuates the effect of PNPLA3 on advanced fibrosis. ${ }^{10}$ Nonetheless, there exists a longitudinal prospective study for rs72613567 prior to the present study that reports a conflicting result, yet the finding can be argued. Despite having a relatively large number of patients $(n=487)$ in their cohort, the patients were diagnosed with chronic viral hepatitis, alcoholic liver disease, or NAFLD-induced portal hypertension who already had advanced chronic liver disease. ${ }^{26}$ In addition, only 56 patients with NAFLD-induced portal hypertension were included in their study. However, the negative findings of Kaplan-Meier survival analysis of patients with different $H S$ D17B13 genotyping status in our cohort may be attributed to the 
small number of events in our study. The mechanism by which loss of HSD17B13 protects against advanced liver disease remains to be elucidated. Nonetheless, growing evidence provides more certainty to the impact of this genetic variant in disease progression in NAFLD patients.

In this study, we also found that serum GGT level is significantly associated with various clinical outcomes. Serum GGT level is a reliable marker of oxidative stress. Haring et al. ${ }^{27}$ reported that elevated GGT levels are associated with an increased risk of mortality in men with hepatic steatosis. In addition, Ruhl and colleagues $^{28}$ reported that in the USA population, elevated GGT is associated with mortality from all causes, liver disease, cancer, and diabetes. A recent study has also found that individuals with repeated elevated GGT levels have an increased risk of developing fatty liver changes. ${ }^{29}$ Nonetheless, serum GGT also contributes to several algorithms for the diagnosis of NAFLD and liver fibrosis such as the fatty liver index (FLI), SteatoTest, and Fibrotest. These findings explain the clinical importance of serum GGT in NAFLD and its role as a predictor of NAFLD outcome.

An interesting finding from our study showed that the rs72613567 heterozygous mutant genotype is associated with a higher incidence of cardiovascular outcome, but not in the homozygous mutant. We speculate that phenotypic consequences of a dominant mutation can be observed in a heterozygous individual carrying one mutant and one wild-type allele. In 1987, Herskowitz $\mathrm{z}^{30}$ described dominant negative mutations as mutations in one allele, when overexpressed, may lead to a structural change in the protein that disrupts the activity of the wild-type gene. For instance, in the context of a dimer $T / T$, if the truncated protein $T A$ renders T/TA and TA/TA dimers inactive, then a heterozygote will produce $25 \% \mathrm{~T} / \mathrm{T}, 50 \% \mathrm{~T} / \mathrm{TA}$ and $25 \% \mathrm{TA} / \mathrm{TA}$, leading to only $25 \%$ of activity with respect to the wild type or homozygous mutant and 50\% activity with respect to the heterozygous. The notion of heterozygous dominant negative mutations causes more severe effects than that does simple null alleles or homozygous alleles of the same gene ${ }^{30,31}$ leads us to believe that the possible mechanism of heterozygous mutations may be more deleterious than that of a truncated protein (TA/TA), which explains the significantly higher incidence of cardiovascular outcome recorded in the rs72613567 T/TA genotype in the present study. However, this novel finding warrants further studies to confirm the impact of rs72613567 on cardiovascular outcomes in patients with NAFLD.

To our knowledge, this study is the first longitudinal cohort study among the Asian population to report a potential protective effect of the protein-truncating HSD17B13 variants on adverse liv- er outcomes in patients with NAFLD. This novel finding may be useful for future pharmacological development of therapeutic gene inhibition by selective enzyme inhibition techniques or could potentially be utilised as a biomarker to predict the development of advanced liver disease. Moreover, our study was able to compare genetic variants among three Asian ethnic groups. This study has several limitations. First, our study had relatively short followup duration in some of our study subjects and hence, a relatively small number of observed events. However, power analysis was conducted prior to the commencement of study to calculate sample sizes and the data available were adequate for statistical significance. Second, we were not able to study the effect of the genetic variants according to ethnic groups due to the even smaller number of observed events when stratified according to ethnicity. Interestingly, there were no liver-related complications among the ethnic Chinese despite the equal proportion of more severe liver disease at baseline compared with that of the other ethnic groups. Third, data on follow-up events were retrieved retrospectively from the medical records; hence, there is a small possibility of missing events while patients seek medical treatment in elsewhere. Due to ethical considerations, liver biopsy was not performed in the controls. Therefore, a list of inclusion and exclusion criteria was used to recruit control subjects, and liver ultrasound was performed to exclude the presence of NAFLD. Lastly, we were unable to follow-up the control subjects and demonstrate a comparison between the NAFLD and non-NAFLD groups in terms of clinical outcomes.

In conclusion, in this two-part study on a cohort of biopsy-proven multi-ethnic Asian NAFLD patients, the loss-of-function variants HSD17B13 rs72613567 and rs6834314 were found to be inversely associated with NASH, specifically among ethnic Chinese, but not among ethnic Malays and Indians. The variants were also found to be independently associated with a lower incidence of liver-related complications. Future studies should examine thoroughly the impact of genetic variants on liver-related outcomes in different ethnic groups, explore the use of genetic variants to predict more severe liver disease, and thereby identify patients for early interventions. We must investigate the underlying mechanism of the pathogenic link between HSD17B13 and adverse liverrelated outcomes in NAFLD, and explore the therapeutic potential of these genetic variants.

\section{Authors' contribution}

RM, SMZ and ZM designed the research study. YWT, SMZ, WKC, HLT established cohort and collected the clinical data for 
Yi-Wen Ting, et al.

patients. YWT, ASYK, SMZ and HLT carried out genotyping. YWT carried out data analysis and documented the findings. YWT, WKC and SMZ wrote the manuscript. RM, SMZ, WKC and YFP provided critical inputs to the manuscripts. The final version of the manuscript has been read and approved by all the authors.

\section{Acknowledgements}

The authors thank all the participants and staff of the UMMC for the assistance during patient's recruitment. This study was supported by University Malaya Research Grant BK077-2016.

\section{Conflicts of Interest}

The authors have no conflicts to disclose.

\section{SUPPLEMENTARY MATERIAL}

Supplementary material is available at Clinical and Molecular Hepatology website (http://www.e-cmh.org).

\section{REFERENCES}

1. Chalasani N, Younossi Z, Lavine JE, Charlton M, Cusi K, Rinella M, et al. The diagnosis and management of nonalcoholic fatty liver disease: practice guidance from the American association for the study of liver diseases. Hepatology 2018;67:328-357.

2. Younossi Z, Stepanova M, Ong JP, Jacobson IM, Bugianesi E, Duseja A, et al. Nonalcoholic steatohepatitis is the fastest growing cause of hepatocellular carcinoma in liver transplant candidates. Clin Gastroenterol Hepatol 2019;17:748-755.e3.

3. Sookoian S, Pirola CJ. Genetic predisposition in nonalcoholic fatty liver disease. Clin Mol Hepatol 2017;23:1-12.

4. Romeo S, Kozlitina J, Xing C, Pertsemlidis A, Cox D, Pennacchio LA, et al. Genetic variation in PNPLA3 confers susceptibility to nonalcoholic fatty liver disease. Nat Genet 2008;40:1461-1465.

5. Liu YL, Patman $G L$, Leathart JB, Piguet $A C$, Burt $A D$, Dufour JF, et al. Carriage of the PNPLA3 rs738409 C > G polymorphism confers an increased risk of non-alcoholic fatty liver disease associated hepatocellular carcinoma. J Hepatol 2014;61:75-81.

6. Abul-Husn NS, Cheng X, Li AH, Xin Y, Schurmann C, Stevis P, et al. A protein-truncating HSD17B13 variant and protection from chronic liver disease. N Engl J Med 2018;378:1096-1106.

7. Pirola CJ, Garaycoechea M, Flichman D, Arrese M, San Martino J, Gazzi C, et al. Splice variant rs72613567 prevents worst histologic outcomes in patients with nonalcoholic fatty liver disease. J Lipid
Res 2019;60:176-185.

8. Ma Y, Belyaeva OV, Brown PM, Fujita K, Valles K, Karki S, et al. 17beta hydroxysteroid dehydrogenase 13 is a hepatic retinol dehydrogenase associated with histological features of nonalcoholic fatty liver disease. Hepatology 2019;69:1504-1519.

9. Gellert-Kristensen H, Nordestgaard BG, Tybjaerg-Hansen A, Stender S. High risk of fatty liver disease amplifies the alanine transaminaselowering effect of a HSD17B13 variant. Hepatology 2020;71:56-66.

10. Seko Y, Yamaguchi K, Tochiki N, Yano K, Takahashi A, Okishio S, et al. Attenuated effect of PNPLA3 on hepatic fibrosis by HSD17B13 in Japanese patients with non-alcoholic fatty liver disease. Liver Int 2020;40:1686-1692.

11. World Health Organization. Regional Office for the Western Pacific. The Asia-Pacific perspective : redefining obesity and its treatment. Sydney: Health Communications Australia, 2000.

12. Expert Panel on Detection, Evaluation, Treatment of High Blood Cholesterol in Adults. Executive summary of the third report of the national cholesterol education program (NCEP) expert panel on detection, evaluation, and treatment of high blood cholesterol in adults (Adult Treatment Panel III). JAMA 2001;285:2486-2497.

13. Brunt EM, Kleiner DE, Wilson LA, Belt P, Neuschwander-Tetri BA; NASH Clinical Research Network (CRN). Nonalcoholic fatty liver disease (NAFLD) activity score and the histopathologic diagnosis in NAFLD: distinct clinicopathologic meanings. Hepatology 2011;53:810-820.

14. Kleiner DE, Brunt EM, Van Natta M, Behling C, Contos MJ, Cummings $\mathrm{OW}$, et al. Design and validation of a histological scoring system for nonalcoholic fatty liver disease. Hepatology 2005;41:13131321.

15. Gauderman WJ. Sample size requirements for matched case-contro studies of gene-environment interaction. Stat Med 2002;21:35-50.

16. Goh SC, Ho EL, Goh KL. Prevalence and risk factors of non-alcoholic fatty liver disease in a multiracial suburban Asian population in Malaysia. Hepatol Int 2013;7:548-554.

17. Browning JD, Szczepaniak LS, Dobbins R, Nuremberg P, Horton $J D$, Cohen JC, et al. Prevalence of hepatic steatosis in an urban population in the United States: impact of ethnicity. Hepatology 2004; 40:1387-1395.

18. Sookoian S, Pirola CJ. Meta-analysis of the influence of I148M variant of patatin-like phospholipase domain containing 3 gene (PNPLA3) on the susceptibility and histological severity of nonalcoholic fatty liver disease. Hepatology 2011;53:1883-1894.

19. Chan WK, Bahar N, Razlan H, Vijayananthan A, Sithaneshwar P, Goh KL. Non-alcoholic fatty liver disease in a young multiracial Asian population: a worrying ethnic predilection in Malay and Indian males. Hepatol Int 2014;8:121-127.

20. Zain SM, Mohamed R, Mahadeva S, Cheah PL, Rampal S, Basu RC, et al. A multi-ethnic study of a PNPLA3 gene variant and its associa- 


\section{CLINICAL and MOLECULAR
HEPATOLOGY}

tion with disease severity in non-alcoholic fatty liver disease. Hum Genet 2012;131:1145-1152.

21. Younossi Z, Anstee QM, Marietti M, Hardy T, Henry L, Eslam M, et al. Global burden of NAFLD and NASH: trends, predictions, risk factors and prevention. Nat Rev Gastroenterol Hepatol 2018;15:11-20.

22. Horiguchi Y, Araki M, Motojima K. 17beta-Hydroxysteroid dehydrogenase type 13 is a liver-specific lipid droplet-associated protein. Biochem Biophys Res Commun 2008;370:235-238.

23. Su W, Wang Y, Jia X, Wu W, Li L, Tian $X$, et al. Comparative proteomic study reveals $17 \beta-H S D 13$ as a pathogenic protein in nonalcoholic fatty liver disease. Proc Natl Acad Sci U S A 2014;111:1143711442.

24. Su W, Mao Z, Liu Y, Zhang X, Zhang W, Gustafsson JA, et al. Role of HSD17B13 in the liver physiology and pathophysiology. Mol Cell Endocrinol 2019;489:119-125.

25. Wang P, Wu CX, Li Y, Shen N. HSD17B13 rs72613567 protects against liver diseases and histological progression of nonalcoholic fatty liver disease: a systematic review and meta-analysis. Eur Rev Med Pharmacol Sci 2020;24:8997-9007.
26. Scheiner $B$, Stättermayer $A F$, Schwabl $P$, Bucsics $T$, Paternostro $R$, Bauer D, et al. Impact of HSD17B13 rs72613567 genotype on hepatic decompensation and mortality in patients with portal hypertension. Liver Int 2020;40:393-404.

27. Haring R, Wallaschofski H, Nauck M, Dörr M, Baumeister SE, Völzke $H$. Ultrasonographic hepatic steatosis increases prediction of mortality risk from elevated serum gamma-glutamyl transpeptidase levels. Hepatology 2009;50:1403-1411.

28. Ruhl CE, Everhart JE. Elevated serum alanine aminotransferase and gamma-glutamyltransferase and mortality in the United States population. Gastroenterology 2009;136:477-485.e11.

29. Fujii H, Doi H, Ko T, Fukuma T, Kadono T, Asaeda K, et al. Frequently abnormal serum gamma-glutamyl transferase activity is associated with future development of fatty liver: a retrospective cohort study. BMC Gastroenterol 2020;20:217.

30. Herskowitz I. Functional inactivation of genes by dominant negative mutations. Nature 1987;329:219-222.

31. Strachan TR, Andrew P. Human Molecular Genetics. 4th ed. New York: Garland Science Taylor and Francis Group, 2011. 